\title{
Water and Methane in Shale Rocks: Flow Pattern Effects on Fluid Transport and Pore Structure
}

\author{
Tuan A. Ho and Alberto Striolo* \\ University College London \\ Department of Chemical Engineering \\ Torrington Place, London WC1E 7JE, United Kingdom \\ * Author to whom all correspondence should be addressed: \\ Email: $\quad$ a.striolo@ucl.ac.uk \\ Phone: $\quad$ +4402076793826 \\ Fax: $\quad$ +4402073832348
}

\begin{abstract}
Using molecular dynamics simulations we study the two-phase flow of water and methane through slit-shaped nano-pores carved from muscovite. The simulations are designed to investigate the effect of flow patterns on the fluids transport and on the pore structure. The results indicate that the Darcy's law, which describes a linear relation between flow rate and pressure drop, can be violated when the flow pattern is altered. This can happen when the driving force, i.e., the pressure drop, increases above a pore-size dependent threshold. Because the system considered here contains two phases, when the fluid structure changes, the movement of methane with respect to that of water changes, leading to the violation of the Darcy's law. Our results illustrate the importance of the capillary force, due to the formation of water bridges across the model pores, not only on the fluid flow, but also on the pore structure, in particular its width. When the water bridges are broken, perhaps because of fast fluid flow, the capillary force vanishes leading to significant pore expansion. Because muscovite is a model for illite, a clay often found in shale rocks, these results advance our understanding regarding the mechanism of water and gas transport in tight shale gas formations.
\end{abstract}




\section{Introduction}

In just a few decades, shale gas has become one of the most important energy resources for the USA, with significant contributions to the natural gas production in the country. ${ }^{1}$ The economical success related to shale gas production has generated interest worldwide, and research has been initiated in many countries to explore the vast shale formations present throughout the world. Research is needed because shale formations are characterized by small porosity, compared to, e.g., sandstone formations, as they are composed of pores with size ranging from 1 to $200 \mathrm{~nm}^{2}$ Because of these features, the permeability of shale rocks can be as low as $1-100$ nanodarcy, (for comparison, the permeability of sandstone is of the order of 1-10 millidarcy). Hydraulic fracturing is practiced to increase the extremely low permeability of shale rocks to enable the economic production of gas, and sometimes oil. ${ }^{3}$ Because water can both be injected and be present naturally in some shale formations, one complicating characteristic is that water and natural gas can co-exist within the pores, leading to the possibility that two-phase flow occurs through the extremely narrow pores of shale formations. ${ }^{4}$ The interactions between water, gas, and shale rocks within such tight environment can lead to capillary forces and other surface phenomena. Understanding these interactions and how they affect the fluid transport is crucial to design effective stimulation practices and optimal gas production strategies, as well as for reducing the environmental impact of shale gas. ${ }^{3,5}$ Building on the results obtained by those scientists devoted to study the behaviour of fluids in narrow pores (i.e., the adsorption community), it is our goal to better understand the mechanism of fluid migration, in particular when two phases form, through shale formations using various modeling approaches.

Two-phase flow is a common problem encountered in many practical applications in chemical engineering, oil recovery, food industry, and bio- technologies. ${ }^{6}$ Although 
much is known about two-phase flow in macro- and micro-scale channels, ${ }^{7}$ little is known about it in nano-channels due to the technical difficulty in fabricating and manipulating nano-devices, in measuring the flow rate in such systems, and in visualizing the flow pattern. ${ }^{8}$ As mentioned above, it is expected that the two-phase flow in nano-channels will differ compared to that in wider channels because capillary and surface forces could generate unexpected effects.

The Darcy's law is often used to describe macroscopically the fluid flow through a porous material: $Q=-\frac{k A}{\eta} \frac{d P}{d x}$, where $\mathrm{Q}$ is the flow rate, A is the cross-sectional area, $\eta$ is the viscosity of the fluid, $\mathrm{k}$ is the permeability, and $\frac{d P}{d x}$ is the pressure drop. ${ }^{9}$ According to the Darcy's law, the flow rate is linearly proportional to the driving force (i.e., the pressure drop). When two phases are present, the flow rate of each phase is linearly proportional to the driving force ${ }^{10}$ only if one phase does not interfere with the flow of the other. ${ }^{11}$ In this case, one phase effectively reduces the pore area available for the flow of the other. However, when fluid transport occurs in nano-pores, the enhanced complexity due to the combination of the interactions between the fluids, the significance of viscous and capillary forces, and the pore morphology might cause the Darcy's law to no longer provide accurate predictions of fluid flow. ${ }^{10,12,13}$ Some of these effects, in particular the two-phase flow pattern and its dependence on the pore morphology, have been studied extensively in micro- and macro-channels. ${ }^{7,14,15}$ Similar studies are prohibitive at the at nano-scale because of multiple technical challenges. ${ }^{8}$ Recently, Wu et al. ${ }^{4,8}$ used optical imaging to study single and two-phase pressure-driven flows in silicon nitride nano-channels of width $100 \mathrm{~nm}$. They concluded that the linear correlation between flow rate and pressure drop was upheld for single-phase flow, and they reported three different flow patterns including single, annular, and stratified for two-phase flows. Perhaps molecular 
modeling can help better understanding the two-phase flow mechanisms in nanochannels.

In this work we employ molecular dynamics simulations to study the flow of water and methane inside slit-shaped nano-channels obtained from muscovite. Muscovite is a popular substrate because of its perfect cleavage, which allows the creation of large surfaces that are atomically smooth. ${ }^{16}$ We chose muscovite because it has similar structure to illite, ${ }^{17}$ a common clay in sedimentary rock environments, including the shale formations ${ }^{18}$ found in the Marcellus and Barnett regions. ${ }^{19,20}$

In the remainder of the paper we first present some details regarding the model substrate and the algorithms implemented for our simulations, we then discuss the results and how they are pertinent to (i) the two-phase flow in narrow pores and (ii) the pore deformation due to fluid flow and imposed pressure.

\section{Simulation details}

In Figure 1 we report a schematic representation of one of our simulated systems. For all simulations, 1800 water and 600 methane molecules are placed inside the slitshape pore obtained from muscovite. Muscovite is a phyllosilicate mineral ${ }^{21,22}$ with TOT structure: an Al-centred octahedral sheet is sandwiched between two Si-centred tetrahedral sheets, in which one $\mathrm{Al}$ atom substitutes one out of every four Si atoms. An interlayer of potassium ions balances the negative charge due to the $\mathrm{Al}$ substitution. The potassium interlayer holds the TOT layers together via electrostatic interactions. ${ }^{23}$ In our model, the muscovite substrate spans $6.2 \mathrm{~nm}$ along the $\mathrm{X}, 5.5 \mathrm{~nm}$ along the $\mathrm{Y}$, and $1.96 \mathrm{~nm}$ along the $\mathrm{Z}$ directions. The atoms in the muscovite mineral, water, and methane are simulated by implementing the CLAYFF, ${ }^{24} \mathrm{SPC} / \mathrm{E},{ }^{25}$ and TraPPE $^{26}$ force fields, respectively. The atomic coordinates used to describe the 
muscovite sample in our simulations, as well all interaction parameters as implemented in the GROMACS simulation package, are provided as Supporting Information. In all cases, the temperature is kept constant at $300 \mathrm{~K}$. The pore-pressure is either $75 \mathrm{MPa}$ or $250 \mathrm{MPa}$, conditions usually implemented in laboratory studies for rock permeability using the triaxial-test method. ${ }^{27,28}$

Periodic boundary conditions are applied in all directions for all simulations. Therefore, the muscovite substrate is infinitely long in $\mathrm{X}$ and $\mathrm{Y}$ directions. Following the methods implemented in our prior studies, in the $\mathrm{Z}$ direction a large vacuum volume (of at least $7.5 \mathrm{~nm}$ in thickness) is added above the muscovite layer to minimize unphysical effects due to interactions between periodic images of the simulated system. ${ }^{29}$ The equations of motion are integrated using the GROMACS simulation package, version 4.0.7, with the time step of $1 \mathrm{fs}$.

\section{Pore-pressure control}

To obtain the desired pore-pressure, we apply a force along the $\mathrm{Z}$ direction onto the top surface. The pore pressure is calculated dividing the applied force by the XY simulation box area. Both muscovite surfaces are described as rigid bodies. The top surface is kept rigid in $\mathrm{X}$ and $\mathrm{Y}$ directions, but is free to move along the $\mathrm{Z}$ direction. The bottom surface is kept rigid (see Figure 1). We start from an initial configuration in which the pore size is $5 \mathrm{~nm}$ (shown in Figure 1). We apply a force $\mathrm{F}_{1}$ as necessary to apply $250 \mathrm{MPa}$ to the pore. During this simulation the pore shrinks from $5 \mathrm{~nm}$ to

\section{$2.65 \mathrm{~nm}$.}

To prepare the $75 \mathrm{MPa}$ pore system we follow two simulation protocols. In the first protocol, i.e., the 'compression' protocol, we start from the initial configuration in which the pore width is $5 \mathrm{~nm}$ (system of Figure 1) and apply a force $F_{2}\left(F_{2}<F_{1}\right)$ onto 
the top surface. As the simulation progresses, the pore shrinks to a width, discussed later, that is wider than the $2.65 \mathrm{~nm}$ achieved for the $250 \mathrm{MPa}$ pore-pressure system. In the second protocol, i.e., the 'expansion' protocol, we use as initial configuration the system of width $2.65 \mathrm{~nm}$ (the $250 \mathrm{MPa}$ pore-pressure system) and we reduce the applied force from $F_{1}$ to $F_{2}$. As the applied pressure is reduced, the pore widens.

All of the pore-pressure simulations are conducted for 30ns. A constant pore size is usually obtained after $6 \mathrm{~ns}$.

\section{Poiseuille flow simulations}

The two-phase flow inside the muscovite nanopore is studied by conducting Poiseuille flow simulations, using a non-equilibrium approach. These simulations are initiated from the final configurations of the pore-pressure control simulations described above. The simulation conditions are the same as those applied above, i.e., the surfaces are treated as rigid bodies and we continue to apply the force along the $\mathrm{Z}$ direction, however, a constant acceleration is applied along the $\mathrm{X}$ direction of the simulation box, ranging from 0.02 to $0.08 \mathrm{~nm} / \mathrm{ps}^{2}$, to all water and methane molecules within the pore. Although these applied accelerations are meant to mimic a pressuredriven flow, ${ }^{30-32}$ they are too high to be realistic (note that the corresponding pressure drop across the pore in our calculations ranges approximately from $1.5 \times 10^{16} \mathrm{~Pa} / \mathrm{m}$ to $\left.6 \times 10^{16} \mathrm{~Pa} / \mathrm{m}\right)$; this is due to computing power limitations. ${ }^{33,34}$ The simulations are conducted until steady states (i.e., constant velocity profiles for the fluid inside the pore) are obtained. At steady states viscous and friction forces balance the external force applied to the fluid molecules. The Poiseuille flow simulations are conducted for $30 \mathrm{~ns}$. The steady state is usually obtained after $10 \mathrm{~ns}$.

Two approaches are usually implemented to control the temperature in nonequilibrium simulations such as those just described: the thermostat is coupled to all 
fluid atoms in the system, or the thermostat is coupled only to the surfaces. ${ }^{35}$ In the latter case, the confined fluid molecules exchange heat with the wall during the course of the simulation. ${ }^{36}$ For the former case, it is essential to subtract the nonzero streaming velocity in the direction of the flow when calculating the kinetic energy. However, because the streaming velocity is un-known, only the velocity component perpendicular to the flow direction is usually thermostatted. ${ }^{37}$ In our simulations, since the streaming velocity is very small compared to the thermal velocity, we include the streaming velocity in our temperature calculations. This will not result in significant error because small streaming velocity contributes only a tiny fraction of the total kinetic energy. ${ }^{38,39}$

\section{Results and Discussion}

Pore-pressure 250MPa

In Figure $2 \mathrm{~A}$ we report a simulation snapshot representing the final configuration of the $250 \mathrm{MPa}$ pore-pressure control simulation. The simulation snapshot confirms that we are in the presence of a two-phase system. Visual inspection shows that water preferentially wets the muscovite surfaces and that a bridge of water molecules is formed between the two pore surfaces. Methane molecules form one gas bubble that is trapped within water. From this equilibrium configuration we initiate the flow simulations by applying a constant acceleration to the fluid molecules along the $\mathrm{X}$ direction. The average velocities of water (filled circles) and methane (empty circles) obtained at steady states as a function of the applied acceleration are presented in Figure 2B. The results indicate that the average velocity of water increases linearly as the applied acceleration increases, which is consistent with the Darcy's law, as the applied acceleration is the driving force for the flux of water. The results obtained for methane differ significantly from those just described for water. In particular, the 
average velocity for methane increases linearly when the acceleration increases from 0.02 to $0.05 \mathrm{~nm} / \mathrm{ps}^{2}$, and then again from 0.06 to $0.08 \mathrm{~nm} / \mathrm{ps}^{2}$. As the acceleration increase from 0.05 to $0.06 \mathrm{~nm} / \mathrm{ps}^{2}$, the Darcy's law is violated, as a step increase of the methane velocity is observed. It is also worth pointing out that, even though both below $0.05 \mathrm{~nm} / \mathrm{ps}^{2}$ and above $0.06 \mathrm{~nm} / \mathrm{ps}^{2}$ the relationship between average velocity and applied acceleration is linear, the slopes of the lines differ, suggesting that the effective permeability of the pore is larger at higher applied accelerations. As we will show below, the results in Figure 2 suggest that the Darcy's law can be used to describe the two-phase flow in nano-channels only if there is no change in flow pattern. They also suggest, perhaps more importantly, that the permeability of the porous material depends strongly on the structure of the confined fluid, which can change upon variations in external stimuli, including applied pressure drops. Visualization of the flow patterns, presented in Figure 3, provides justification for these insights, in particular concerning the breakdown of the Darcy's law when the acceleration increases from 0.05 to $0.06 \mathrm{~nm} / \mathrm{ps}^{2}$.

In Figure 3 we present the flow patterns inside the pore of Figure 2 when the applied acceleration is $0.05 \mathrm{~nm} / \mathrm{ps}^{2}$ (left) and $0.06 \mathrm{~nm} / \mathrm{ps}^{2}$ (right). In all cases, the flow occurs along the $\mathrm{X}$ direction, and the snapshots are obtained after steady states conditions are established. The results presented in the left panels show the water bridge, formed between the two surfaces, which spans the entire length of the pore along the $\mathrm{Y}$ direction. This flow pattern is observed when the applied acceleration is $0.05 \mathrm{~nm} / \mathrm{ps}^{2}$ or smaller. This pattern is consistent with the 'slug flow' observed for two-phase flow at larger length scales when the gas phase exists as a large bubbles separated from each other by liquid 'slugs'. ${ }^{40}$ 
When the acceleration increases to $0.06 \mathrm{~nm} / \mathrm{ps}^{2}$, the flow pattern changes, as shown in the right panels of Figure 3. The water bridge between the two surfaces is still present, but it no longer spans the entire length of the pore along the $\mathrm{Y}$ direction and resembles a water 'pillar' surrounded by methane. As a consequence, water molecules reduce the flow area available to methane, but they do not slow its flow. In other words, when the acceleration is high enough the gas phase breaks through the liquid phase. ${ }^{41}$ Macroscopically, this phenomenon is expected to occur when the pressure difference between two phases is larger than the capillary pressure given by YoungLaplace equation $\Delta P_{c}=2 \gamma \cos \theta / h$, where $\gamma$ is the surface tension at water/gas interface and $\theta$ is the contact angle. ${ }^{42}$ The flow pattern just described does not change when the acceleration increases from 0.06 to $0.08 \mathrm{~nm} / \mathrm{ps}^{2}$. Because within the conditions of Figures 2 and 3 when the flow pattern changes the pore size does not change, our results suggest that the violation of the Darcy's law observed for methane when the applied acceleration increases from 0.05 to $0.06 \mathrm{~nm} / \mathrm{ps}^{2}$ is due to the change in flow pattern. Within this range of conditions the flow of water continues to obey the Darcy's law. We also point out that the flow pattern change just discussed is irreversible. In other words, even if we reduce the applied acceleration from 0.06 to any value below $0.05 \mathrm{~nm} / \mathrm{ps}^{2}$, the flow pattern remains the one described in the right panels of Figure 3, and the one described on the left panels of the figure is not reestablished. This is probably evidence of the possibility that long-lived metastable states can strongly affect two-phase fluid flow through nano-pores.

To better understand how the flow pattern affects the flow of water and methane through the slit-shaped muscovite nanopore we present in Figure 4A the velocity profiles of water (filled symbols) and methane (empty symbols) as a function of the 
position within the pore when the acceleration is $0.05 \mathrm{~nm} / \mathrm{ps}^{2}$ (circles) and $0.06 \mathrm{~nm} / \mathrm{ps}^{2}$ (triangles). At the acceleration of $0.05 \mathrm{~nm} / \mathrm{ps}^{2}$ (circles) the velocity profile of water and that of methane suggest that at the pore center methane travels at the same speed as water does (note that very few methane molecules are found near the surface). This suggests that, effectively, the water bridge blocks the methane transport in the direction of flow. At the higher applied accelerations, the results in Figure 4A show that, at every position within the pore, methane travels much faster than water does. This happens because the water bridge no longer blocks methane transport, as it no longer spans the entire width of the nano-pore. In this configuration methane molecules can move through the pore free from physical interactions with water. The effect of the change in flow pattern becomes more evident when we compare the average velocity of methane to that of water inside the pore (Figure 4B). For example, at low accelerations $\left(0.02\right.$ to $\left.0.05 \mathrm{~nm} / \mathrm{ps}^{2}\right)$ the ratio between the average velocity of methane and that of water is $\sim 2$, while at higher accelerations $\left(0.06\right.$ to $\left.0.08 \mathrm{~nm} / \mathrm{ps}^{2}\right)$ this ratio is $\sim 4.3$. Note that at the applied acceleration of $0.05 \mathrm{~nm} / \mathrm{ps}^{2}$, despite the fact that the velocity of water and that of methane are the same in the middle of the pore (see Figure 4A), the average velocity of methane is twice the average velocity of water (see Figure 4B). This is because water wets the muscovite surface, and the water molecules in the region near the solid surface are effectively not moving along the direction of motion.

\section{Pore-pressure 75MPa}

The results discussed in Figure 2 strongly depend on the presence of the water bridge and on the flow pattern within the muscovite pore. Building on prior simulation studies for water in clay pores, ${ }^{43,44}$ we expect that the stability of the water bridge will 
depend on the amount of water present within the pore, on the pore size, and on the pore pressure. To test this possibility we conducted simulations reducing the pore pressure from $250 \mathrm{MPa}$ to $75 \mathrm{MPa}$. In Figure 5 we show how the pore width changes as a function of time when the applied pressure is instantaneously changed from low to $75 \mathrm{MPa}$ (compression protocol, filled circles), and when the applied pressure is reduced from $250 \mathrm{MPa}$ to $75 \mathrm{MPa}$ (expansion protocol, empty circles). In the compression protocol the pore width decreases from $5 \mathrm{~nm}$ to $3.58 \mathrm{~nm}$, while in the expansion one the pore width increases from $2.65 \mathrm{~nm}$ to $3.19 \mathrm{~nm}$. These results indicate that starting from two different initial configurations, we obtain two stable configurations (insets $\mathrm{A}$ and $\mathrm{B}$ ) that, although characterized by the same pore pressure, are $0.4 \mathrm{~nm}$ different in width. Analysis of the simulation snapshots (insets) show that the fluid molecules assume different structures within the system: in the configuration presented in the inset $\mathrm{A}$, water molecules accumulate near the solid surfaces while methane remains in the pore center. In the configuration presented in the inset $\mathrm{B}$, water molecules form a bridge between the two solid surfaces. The resultant capillary force brings the two pore surfaces closer by $0.4 \mathrm{~nm}$ compared to when the bridge is not present. No force balance has been conducted to explain this difference. However, a qualitative investigation of the total energy of both compression and expansion systems (results not shown) indicates that the configuration shown in inset B is more stable than that depicted in inset $\mathrm{A}$, suggesting that the capillary force is essential in determining the stable pore structure at the nanoscale.

From the last configurations shown in insets $\mathrm{A}$ and $\mathrm{B}$ for the pore at $75 \mathrm{MPa}$ we initiate flow simulations. The results show that the imposed flow does not change the fluid distribution within the pore when the simulations start from the structure shown 
in inset A for all accelerations applied, which is not surprising. In this case our results are consistent with the annular two-phase flow described in micro-channels. The correspondent average velocity along the $\mathrm{X}$ direction for water (empty circles) and methane (empty triangles) during the simulated two-phase flow are shown in Figure 6A. The results suggest that the average velocities of both water and methane increase linearly for the whole range of acceleration studied, which is consistent with the Darcy's law. The ratio between the average velocity of methane and that of water (empty circles, Figure 6B) is $\sim 12$.

When we simulate the fluid flow starting from the configuration presented in the inset B of Figure 5, our results show that the flow pattern changes over time. When the imposed acceleration is in the range from $0.02 \mathrm{~nm} / \mathrm{ps}^{2}$ to $0.06 \mathrm{~nm} / \mathrm{ps}^{2}$, the water bridge remains, but it becomes thinner as the simulation progresses. One macroscopic consequence of this result is that the pore width slightly increases over time (see filled triangles in Figure 5). When the applied acceleration is increased further to 0.07 and $0.08 \mathrm{~nm} / \mathrm{ps}^{2}$, the water bridge vanishes, causing the expansion of the pore from $3.19 \mathrm{~nm}$ to $3.59 \mathrm{~nm}$ (empty triangles in Figure 5). In other words, when the applied acceleration is large enough, the fluid structure within the pore changes from that pictured in inset $\mathrm{B}$ to that in inset $\mathrm{A}$. The correspondent average velocity along the $\mathrm{X}$ direction for water (filled circles) and methane (filled triangles) during the simulated two-phase flow are shown in Figure 6A. The results indicate that the average velocities of both water and methane increase linearly when the applied acceleration increases from 0.02 to $0.06 \mathrm{~nm} / \mathrm{ps}^{2}$, which is consistent with the Darcy's law. Within this range of applied accelerations, the ratio between the average velocity of methane and that of water (filled circles, Figure 6B) is around 6.4. When the applied acceleration increases to 0.07 and $0.08 \mathrm{~nm} / \mathrm{ps}^{2}$, the Darcy's law is violated, as a step 
increase of the methane average velocity and a slight decrease of the water average velocity are observed. The ratio between the average velocity of methane and that of water increases to 12 (filled circles, Figure 6B), consistent with the results obtained starting the flow simulations from the configuration of inset $\mathrm{A}$ of Figure 5. As described above, when the acceleration increases to $0.07 \mathrm{~nm} / \mathrm{ps}^{2}$, there are major changes in flow pattern and in pore size. Both changes contribute to the step increase of the average velocity of methane. Unexpectedly, the change in flow pattern slightly decreases the average velocity of water, despite of the increase in acceleration. This is because the water in the centre of the pore of inset B of Figure 5 can move faster, even at the smaller acceleration, along the $\mathrm{X}$ direction than the water in the water film near the surface in the inset A of Figure 5. The water molecules at the center of the pore move closer to the surface when the bridge is disrupted, leading to lower average velocity for water molecules.

The results just discussed indicate that the fluid flow can alter the effective interactions between water, methane, and pore surfaces, with effects not only on flow patterns and applications of the Darcy's law, but also on the pore structure. The effects on pore structure are manifested on the pore width, which can increase when the capillary forces due to the presence of water bridges vanish when the bridges disappear. 


\section{Conclusions}

Using molecular dynamics simulation we studied the two-phase flow of water and methane inside slit-shape pores obtained from muscovite. The simulations were designed to investigate the effect of flow patterns on the fluids transport and on the pore structure at the temperature of $300 \mathrm{~K}$ and pore-pressure of either $75 \mathrm{MPa}$ or 250MPa. The results indicate that the Darcy's law is obeyed as long as the flow pattern does not change. When the fluid structure changes, the relative movement of methane with respect to that of water changes, leading to the violation of the Darcy's law. Our results illustrate the importance of capillary forces, which can establish upon the formation of water bridges across the clay pores, not only on the fluid flow, but also on the pore structure, in particular on its width. When the water bridges are broken, perhaps because of fast fluid flow, the capillary force vanish leading to the significant expansion of the pore. Because muscovite is considered a model of illite, a clay often found in the shale formations in the Marcellus and Barnett regions, these results advance our understanding regarding the mechanism of water and gas transport in tight shale gas formations.

\section{Acknowledgements}

Generous allocations of computing time are provided by the Oklahoma Supercomputer Center for Education and Research (OSCER) and by the National Energy Resources Supercomputer Center (NERSC). The authors acknowledge financial support from a Marie Curie Career Initiation Grant and from the Department of Chemical Engineering at University College London. It is a great honour to dedicate this humble contribution to Prof. John M. Prausnitz. His kind words of encouragement have supported and continue to support me throughout all stages of my career and his exemplary intellectual contributions provide everlasting motivation. 


\section{References}

1. Hughes JD. A reality check on the shale revolution. Nature. 2013;494(7437):307-308.

2. Cipolla CL, Lolon E, Mayerhofer MJ. Reservoir Modeling and Production Evaluation in Shale-Gas Reservoirs: International Petroleum Technology Conference; 2009.

3. Yethiraj A, Striolo A. Fracking: What Can Physical Chemistry Offer? The Journal of Physical Chemistry Letters. 2013;4(4):687-690.

4. $\mathrm{Wu} \mathrm{QH}, \mathrm{Bai} \mathrm{BJ}, \mathrm{Ma} \mathrm{YF}, \mathrm{Ok} J \mathrm{~J}$, Neeves KB, Yin XL. Optic Imaging of TwoPhase-Flow Behavior in 1D Nanoscale Channels. Spe J. 2014;19(5):793802.

5. Silin D, Kneafsey TJ. Gas Shale: From Nanometer-scale Observations to Well Modelling. Canadian Society for Unconventional Gas. 2011;CSUG/SPE 149489.

6. Joekar-Niasar V, Hassanizadeh SM. Analysis of Fundamentals of TwoPhase Flow in Porous Media Using Dynamic Pore-Network Models: A Review. Crit Rev Env Sci Tec. 2012;42(18):1895-1976.

7. Shao N, Gavriilidis A, Angeli P. Flow regimes for adiabatic gas-liquid flow in microchannels. Chemical Engineering Science. 2009;64(11):2749-2761.

8. Wu Q, Ok JT, Sun Y, et al. Optic imaging of single and two-phase pressuredriven flows in nano-scale channels. Lab on a Chip. 2013;13(6):11651171.

9. Tanikawa W, Shimamoto T. Comparison of Klinkenberg-corrected gas permeability and water permeability in sedimentary rocks. Int $J$ Rock Mech Min. 2009;46(2):229-238.

10. Ahmadlouydarab M, Liu Z-S, Feng JJ. Relative permeability for two-phase flow through corrugated tubes as model porous media. International Journal of Multiphase Flow. 2012;47(0):85-93.

11. Muskat M, Meres MW. The Flow of Heterogeneous Fluids Through Porous Media. Journal of Applied Physics. 1936;7(9):346-363.

12. Ahmadlouydarab $M$, Liu Z-S, Feng JJ. Interfacial flows in corrugated microchannels: Flow regimes, transitions and hysteresis. International Journal of Multiphase Flow. 2011;37(10):1266-1276.

13. Niessner J, Berg S, Hassanizadeh SM. Comparison of Two-Phase Darcy's Law with a Thermodynamically Consistent Approach. Transp Porous Med. 2011;88(1):133-148.

14. Indraratna $B$, Ranjith P. Laboratory Measurement of Two-Phase Flow Parameters in Rock Joints Based on High Pressure Triaxial Testing. Journal of Geotechnical and Geoenvironmental Engineering. 2001;127(6):530-542.

15. Ranjith PG, Choi SK, Fourar M. Characterization of two-phase flow in a single rock joint. Int J Rock Mech Min. 2006;43(2):216-223.

16. Wang J, Kalinichev AG, Kirkpatrick RJ, Cygan RT. Structure, Energetics, and Dynamics of Water Adsorbed on the Muscovite (001) Surface: A Molecular Dynamics Simulation. The Journal of Physical Chemistry B. 2005;109(33):15893-15905. 
17. White WM. Geochemistry. Oxford, UK: John Wiley \$Sons; 2013.

18. Pevear DR. Illite and hydrocarbon exploration. P Natl Acad Sci USA. 1999;96(7):3440-3446.

19. Tian Y, Ayers WB. Barnett Shale (Mississippian), Fort Worth Basin, Texas: Regional Variations in Gas and Oil Production and Reservoir Properties. Society of Petroleum Engineers: Society of Petroleum Engineers; 2010.

20. Blatt H, Tracy R, Owens B. Petrology: Igneous, Sedimentary and Metamorphic: Freeman; 1996.

21. Wang JW, Kalinichev AG, Kirkpatrick RJ, Cygan RT. Structure, energetics, and dynamics of water adsorbed on the muscovite (001) surface: A molecular dynamics simulation. J Phys Chem B. 2005;109(33):1589315905.

22. Teich-McGoldrick SL, Greathouse JA, Cygan RT. Molecular Dynamics Simulations of Structural and Mechanical Properties of Muscovite: Pressure and Temperature Effects. J Phys Chem C. 2012;116(28):1509915107.

23. Teich-McGoldrick SL, Greathouse JA, Cygan RT. Molecular Dynamics Simulations of Structural and Mechanical Properties of Muscovite: Pressure and Temperature Effects. The Journal of Physical Chemistry C. 2012;116(28):15099-15107.

24. Cygan RT, Liang JJ, Kalinichev AG. Molecular models of hydroxide, oxyhydroxide, and clay phases and the development of a general force field. J Phys Chem B. 2004;108(4):1255-1266.

25. Berendsen HJC, Grigera JR, Straatsma TP. The Missing Term in Effective Pair Potentials. Journal of Physical Chemistry. 1987;91(24):6269-6271.

26. Martin MG, Siepmann JI. Transferable potentials for phase equilibria. 1. United-atom description of n-alkanes. J Phys Chem B. 1998;102(14):25692577.

27. Nara Y, Meredith PG, Yoneda T, Kaneko K. Influence of macro-fractures and micro-fractures on permeability and elastic wave velocities in basalt at elevated pressure. Tectonophysics. 2011;503(1-2):52-59.

28. Tanikawa W, Shimamoto T. comparison of Klinkenberg-corrected gas permeability and water permeability in sedimentary rocks (vol 46, pg 229, 2009). Int J Rock Mech Min. 2009;46(8):1394-1395.

29. Ho TA, Argyris D, Cole DR, Striolo A. Aqueous $\mathrm{NaCl}$ and $\mathrm{CsCl}$ Solutions Confined in Crystalline Slit-Shaped Silica Nanopores of Varying Degree of Protonation. Langmuir. 2012;28(2):1256-1266.

30. Kannam SK, Todd BD, Hansen JS, Daivis PJ. Slip flow in graphene nanochannels. J Chem Phys. 2011;135(14):144701-144701 - 144701144709.

31. Thomas JA, McGaughey AJH, Kuter-Arnebeck O. Pressure-driven water flow through carbon nanotubes: Insights from molecular dynamics simulation. Int J Therm Sci. 2010;49(2):281-289.

32. Gong XJ, Li JY, Zhang H, et al. Enhancement of Water Permeation across a Nanochannel by the Structure outside the Channel. Phys Rev Lett. 2008;101(25):257801-257801 - 257801-257804.

33. Ho TA, Papavassiliou DV, Lee LL, Striolo A. Liquid water can slip on a hydrophilic surface. P Natl Acad Sci USA. 2011;108(39):16170-16175. 
34. Lauga E, MP B, HA S. Handbook of Experimental Fluid Dynamics. New York: Springer; 2007.

35. Travis KP, Gubbins KE. Poiseuille flow of Lennard-Jones fluids in narrow slit pores. J Chem Phys. 2000;112(4):1984-1994.

36. Toton D, Lorenz CD, Rompotis N, Martsinovich N, Kantorovich L. Temperature control in molecular dynamic simulations of nonequilibrium processes. J Phys-Condens Mat. 2010;22(7).

37. Zhu W, Singer SJ, Zheng Z, Conlisk AT. Electro-osmotic flow of a model electrolyte. Phys Rev E. 2005;71(4).

38. Freund JB. Electro-osmosis in a nanometer-scale channel studied by atomistic simulation. J Chem Phys. 2002;116(5):2194-2200.

39. Qiao R, Aluru NR. Ion concentrations and velocity profiles in nanochannel electroosmotic flows. J Chem Phys. 2003;118(10):4692-4701.

40. Rebrov EV. Two-phase flow regimes in microchannels. Theor Found Chem En+. 2010;44(4):355-367.

41. Roydhouse MD, Pradas $\mathrm{M}, \mathrm{Al}$-Rifai $\mathrm{N}$, et al. Operating ranges of gas-liquid capillary microseparators: Experiments and theory. Chemical Engineering Science. 2014;114:30-39.

42. Bruus H. Theoretical Microfluidics. New York: Oxford University Press; 2008.

43. Tambach TJ, Hensen EJM, Smit B. Molecular simulations of swelling clay minerals. J Phys Chem B. 2004;108(23):7586-7596.

44. Rao Q, Xiang Y, Leng YS. Molecular Simulations on the Structure and Dynamics of Water-Methane Fluids between Na-Montmorillonite Clay Surfaces at Elevated Temperature and Pressure. J Phys Chem C. 2013;117(27):14061-14069. 


\section{Figure Captions}

Figure 1. Schematic representation of the initial configuration of our simulated systems. Water (red wireframes) and methane (cyan spheres) are confined in a slitshape muscovite nanopore. Al-centred octahedral, Si-centred tetrahedral, and potassium atoms are presented in green, yellow, and blue colour, respectively. Color online.

Figure 2. Simulation snapshot representing the final configuration of the $250 \mathrm{MPa}$ pore-pressure control simulation (A). Average velocity along the $\mathrm{X}$ direction of water (filled circles) and methane (empty circles) during the two-phase flow within the muscovite nano-pore as a function of the applied acceleration (B). Color online.

Figure 3. Top (top panels) and side (bottom panels) views of the flow patterns inside the pore of Figure 2 (surfaces are removed for clarity) when the applied acceleration is $0.05 \mathrm{~nm} / \mathrm{ps}^{2}$ (left) and $0.06 \mathrm{~nm} / \mathrm{ps}^{2}$ (right). Color online.

Figure 4. Velocity profiles of water (filled symbols) and methane (empty symbols) during the two-phase flow within muscovite pore when the applied accelerations are $0.05 \mathrm{~nm} / \mathrm{ps}^{2}$ (circles) and $0.06 \mathrm{~nm} / \mathrm{ps}^{2}$ (triangles) (A). Ratio between the average velocity of methane and that of water during the two-phase flow as a function of applied acceleration (B).

Figure 5. Pore size as a function of simulation time obtained for $75 \mathrm{MPa}$ compression (filled circles), expansion (empty circles), flow at acceleration of $0.02 \mathrm{~nm} / \mathrm{ps}^{2}$ (filled triangles), and $0.08 \mathrm{~nm} / \mathrm{ps}^{2}$ (empty triangles) simulations.

Figure 6. Average velocity along the $X$ direction of water (circles) and methane (triangles) during the two-phase flow simulated within the muscovite nano-pore described in inset A (empty symbols) and inset B (filled symbols) of Figure 5 a function of the applied acceleration (A). Ratio between the average velocity of methane and that of water during the two-phase flow described in inset B (filled circles) and inset A (empty circles) of Figure 5 as a function of applied acceleration (B). Color online. 
Figure 1

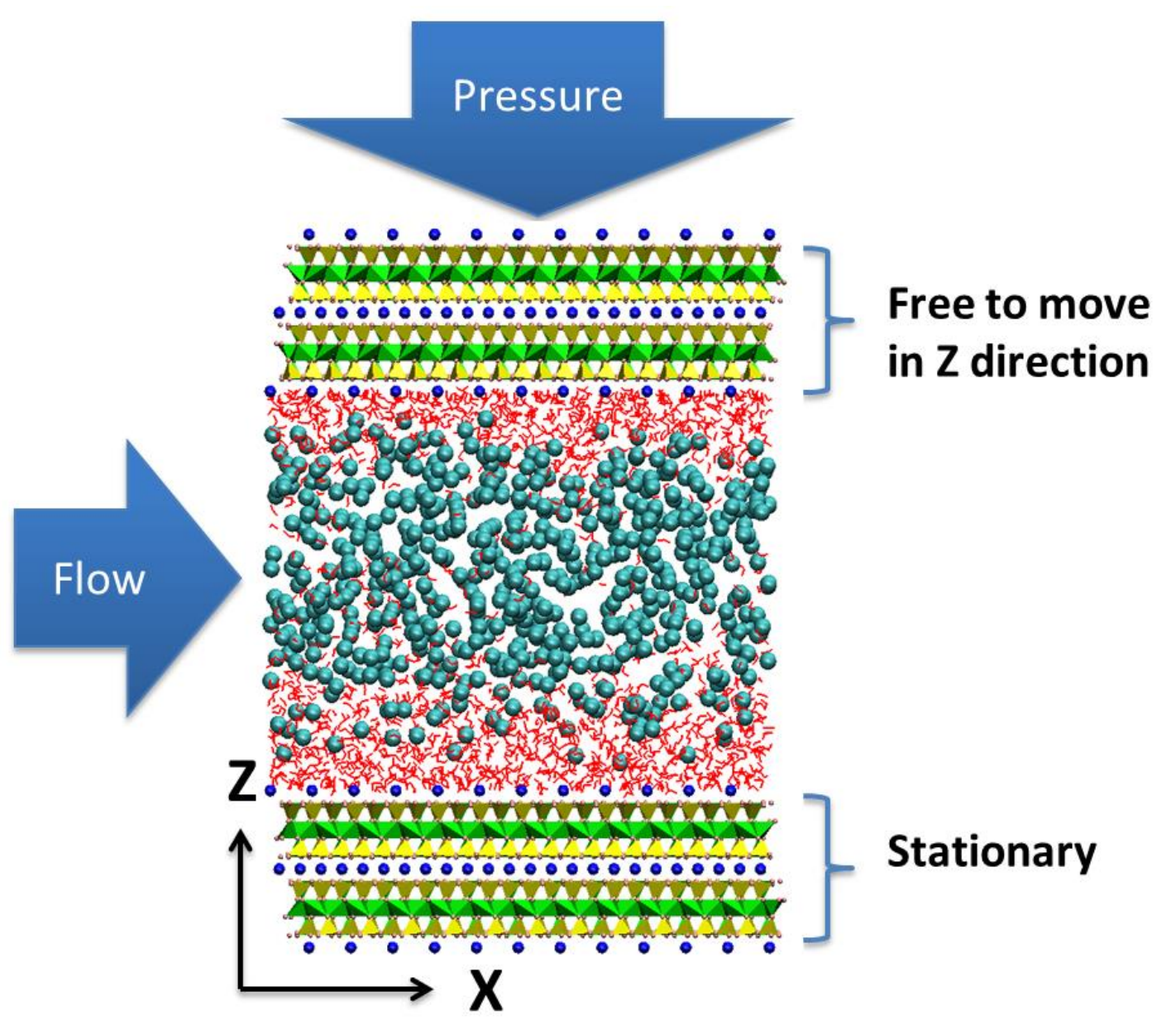


Figure 2
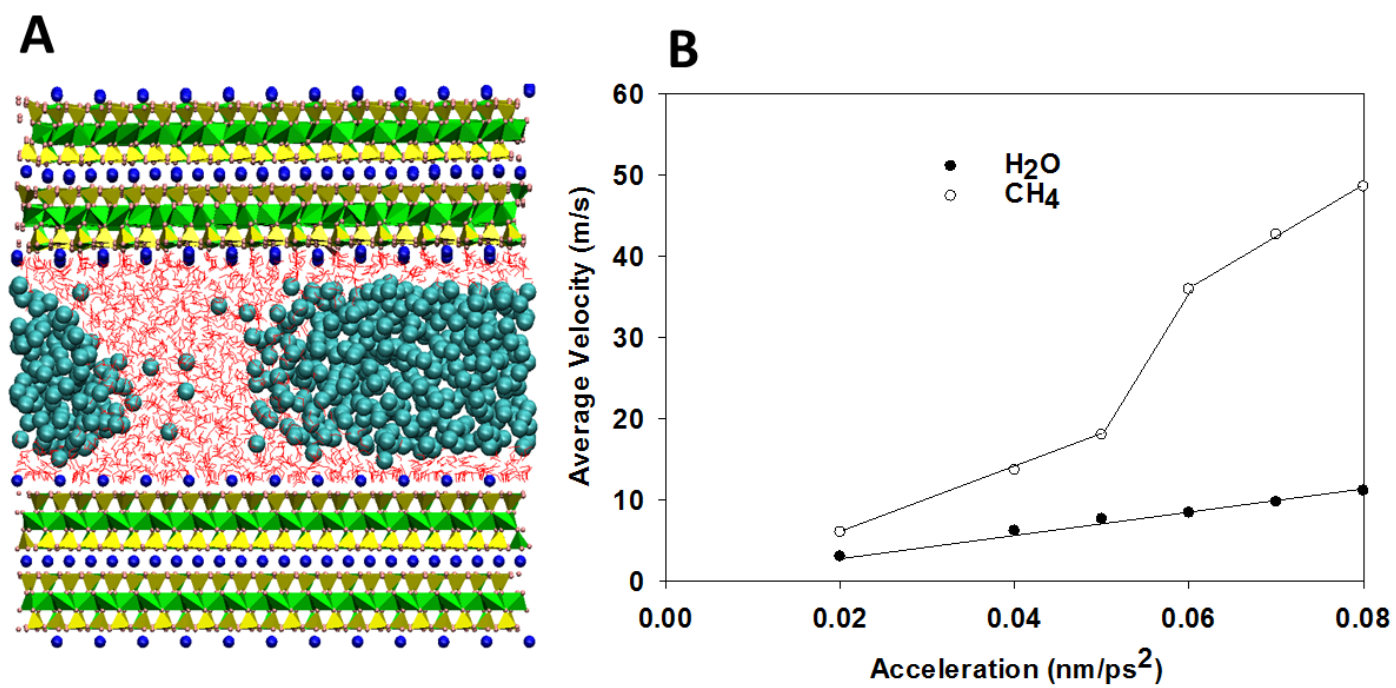
Figure 3
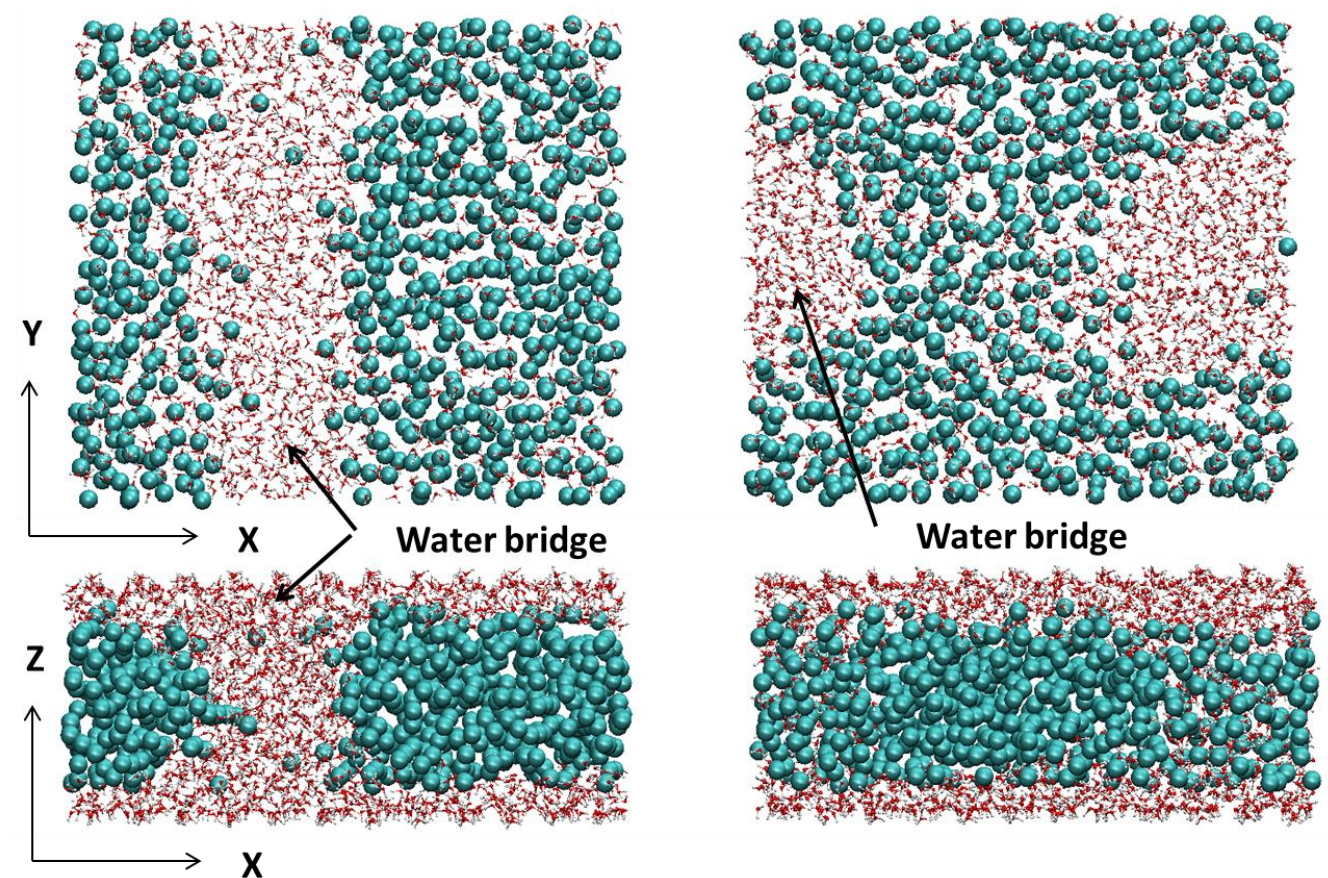
Figure 4
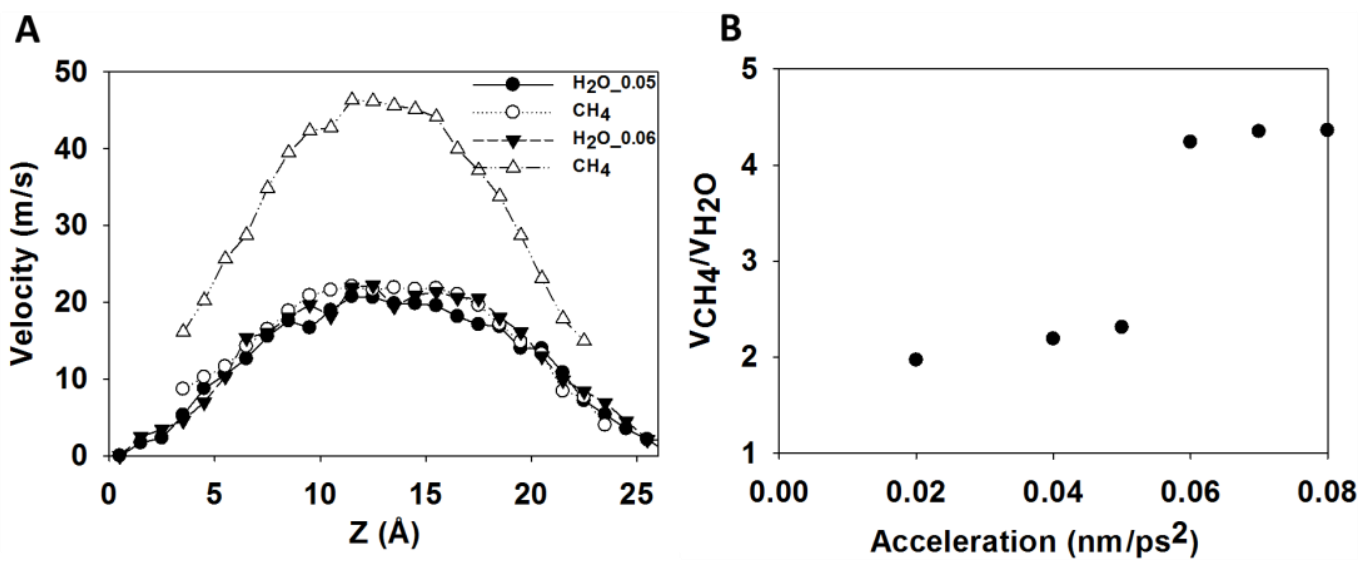
Figure 5

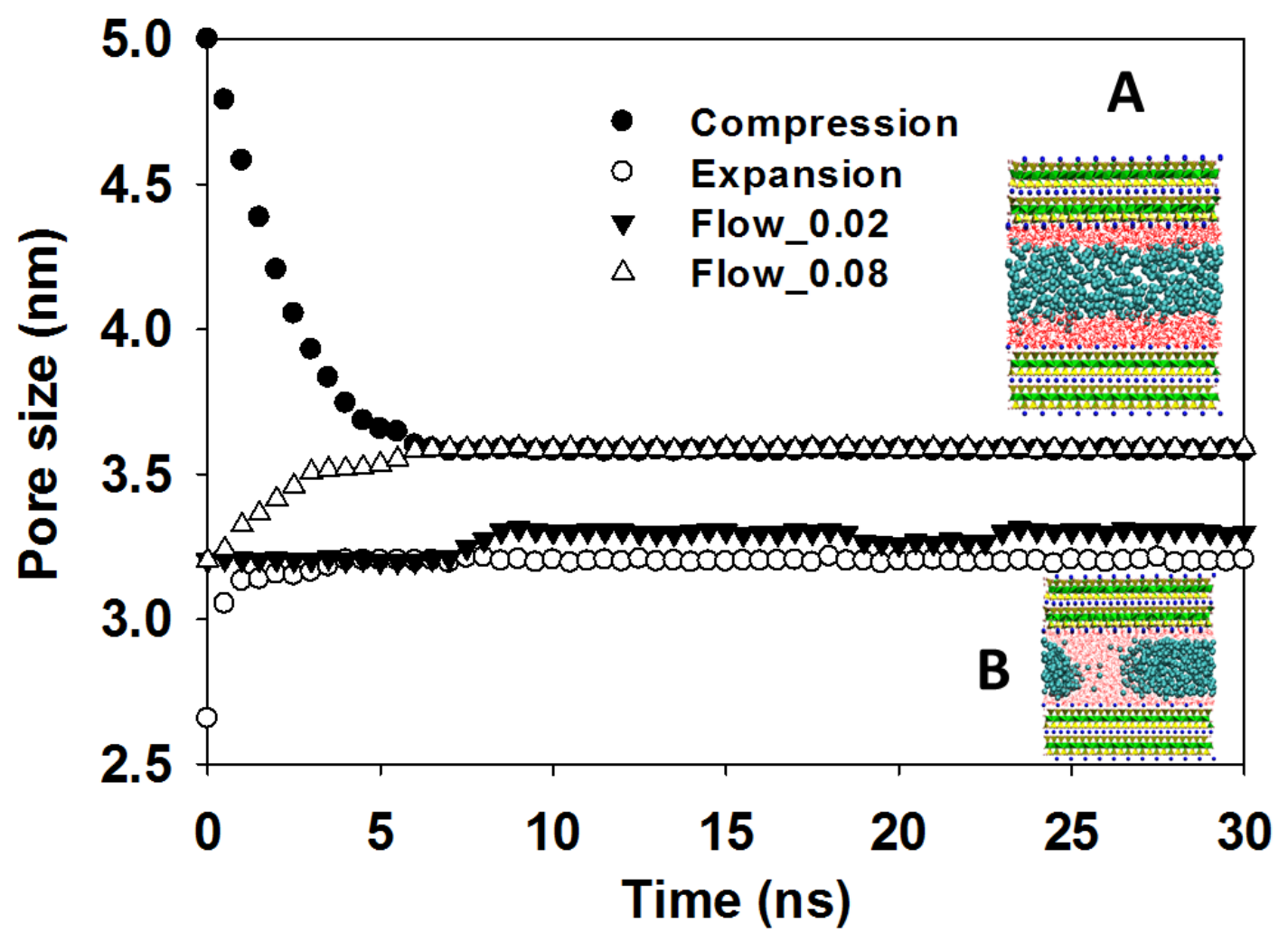


Figure 6

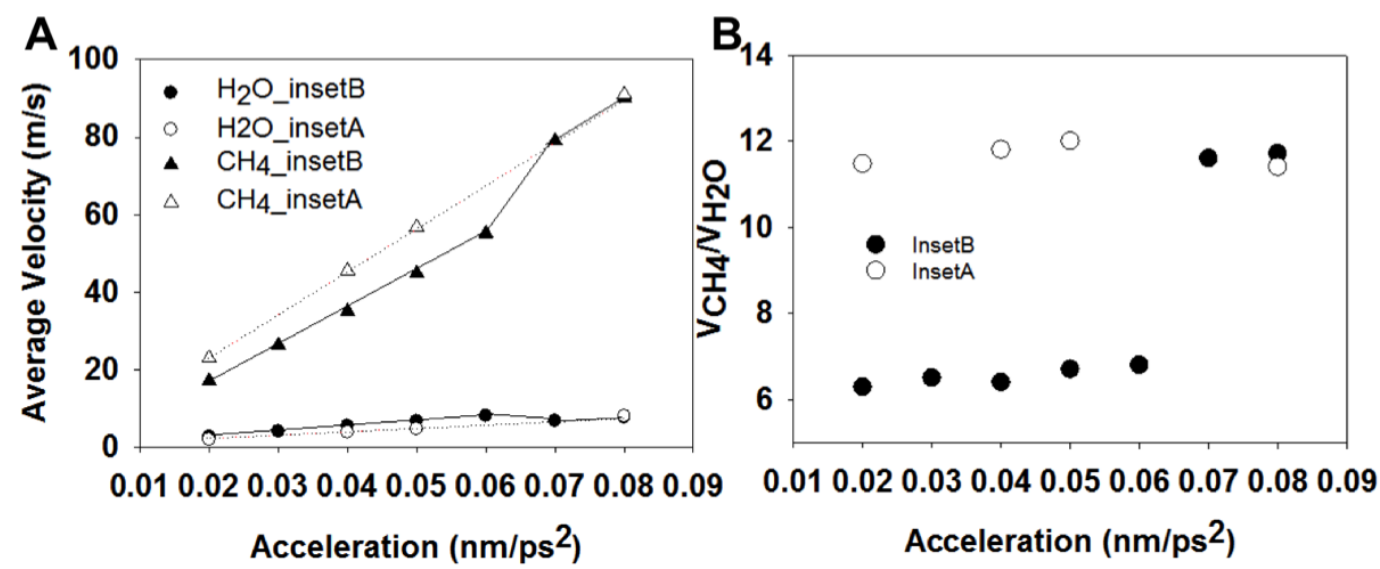

